\title{
Effect of mutual location and the shape of heaters on the stability of thin films flowing over locally heated surfaces
}

\author{
Rong Liu ${ }^{\mathrm{a}, \mathrm{b}, *}$, Oleg A. Kabov ${ }^{\mathrm{c}, \mathrm{d}, \mathrm{e}, *}$ \\ ${ }^{a}$ Key Laboratory of Microgravity (National Microgravity Laboratory), Institute of Mechanics, Chinese Academy of Sciences, Beijing 100190, China \\ ${ }^{\mathrm{b}}$ Chimie-Physique EP-CP165/62, Microgravity Research Center, Université Libre de Bruxelles, Av. Roosevelt 50, Bruxelles B-1050, Belgium \\ ' Institute of Thermophysics, Russian Academy of Sciences, Prosp. Lavrentyev 1, Novosibirsk 630090, Russia \\ ${ }^{\mathrm{d}}$ Centre of Smart Interfaces, Technische Universität Darmstadt, Petersen Str. 30, Darmstadt 64287, Germany \\ ${ }^{\mathrm{e}}$ Tomsk Polytechnic University, Prosp. Lenina 30, Tomsk 634050, Russia
}

\section{A R T I C L E I N F O}

\section{Article history:}

Received 17 October 2012

Received in revised form 8 April 2013

Accepted 19 May 2013

Available online 22 June 2013

\section{Keywords:}

Thin film

Locally heated

Marangoni force

\begin{abstract}
A B S T R A C T
We consider the motion of a liquid film falling down a locally heated planar substrate. Marangoni effect due to the local temperature gradients at the free surface induces a three dimensional interfacial deformation near the vicinity of the heater. The objective of the present paper is to study the influence of streamwise arrangement of the heaters of rectangular shape or with infinite spanwise lengths on the structure of interfacial deformation and the stability of the film. The problem is studied in the framework of longwave theory. We studied the influence of mutual location of heaters on the steady state of the film. As the film is heated by heaters with infinite/finite spanwise lengths, 2D/3D steady states exist. A linear stability analysis is performed with respected to both the 2D and 3D basic state. The results show that the mutual location of heater plays an important role in the stability and transient behavior of the film for 2D steady state. However, the 3D steady state of a film heated by rectangular heaters is linear stable. This means that the 3D steady state is a coherent structure in films heated by rectangular heaters.
\end{abstract}

(c) 2013 Elsevier Ltd. All rights reserved.

\section{Introduction}

The dynamics, stability and rupture of thin liquid films are encountered in many areas of engineering, geophysics, and biophysics, including cooling technologies, nanofluidics, microfluidics, and coating. The effects of thermocapillarity on the instability of flow in thin liquid layers on a solid support have been extensively studied both theoretically and experimentally. For thin films uniformly heated from below, surface-tension-driven Bénard convection can exhibit a longwave primary instability [1] that differs from the classical Marangoni instability with a short wavelength reported by Pearson [2].

In recent years, increasing performance demands in semiconductor technology, including shrinking feature size, increasing transistor density, and faster circuit speeds, have resulted in very high chip power dissipation and heat fluxes. It is also leading to greater non-uniformity of on-chip power dissipation, creating localized, sub-millimeter hot spots, often exceeding $1 \mathrm{~kW} / \mathrm{cm}^{2}$ in heat flux, which can degrade the processor performance and reliability [3]. Similar developments are underway in microwave integrated cir-

\footnotetext{
* Corresponding authors at: Key Laboratory of Microgravity (National Microgravity Laboratory), Institute of Mechanics, Chinese Academy of Sciences, Beijing 100190, China. Tel.: +861082544119.

E-mail addresses: liurong@imech.ac.cn (R. Liu), kabov@itp.nsc.ru (O.A. Kabov).
}

cuits and power amplifier chips, with even higher localized heat fluxes and heat densities. The industrial and technological applications mentioned above involve thin liquid films on locally heated substrates. To avoid the reduction of their performance by film breakdown it is of crucial importance to understand when and why instabilities arise that may result in rupture of the film. Understanding the physical mechanisms of instability and rupture behavior is also highly desirable for the requirement of seeking effective ways to suppress the rupture of locally heated films.

Kabov [4] first studied the motion of the flow in a locally heated film experimentally. Subsequent experimental studies on the instability of locally heated falling films have been reported in Refs. [5-7]. These experimental results reveal the occurrence of novel effects: the competing flows produce a horizontal band of increased film thickness at the upper edge of the heater ('horizontal bump'), which may become unstable and develop rivulets periodic in the direction transverse to the flow. For more details of the phenomenon of 'horizontal bump' and 'rivulet structures', we refer the reader to the review article by Kabov [8].

Skotheim et al. [9] applied longwave theory to study the instability with respect to 2D stead basic state of a falling film over a locally heated substrate. The authors derived a Benney like equation to describe the nonlinear evolution of the film, in which the inertial terms are neglected. Their results show that a rivulet instability occurs with a finite transverse wavelength. Moreover, the calculated film 


\section{Nomenclature}

$\begin{array}{ll}B i & \text { Biot number } \\ B o & \text { Bond number } \\ d & \text { distance between two heaters } \\ \mathbf{g} & \text { vector of gravitational acceleration } \\ g & \text { gravitational acceleration } \\ h & \text { film thickness } \\ H & \text { far upstream film thickness } \\ \hat{H} & \text { amplitude of the normal mode disturbance of the film } \\ & \text { thickness } \\ k & \text { wavenumber } \\ \boldsymbol{L} & \text { linear operator of the stability problem } \\ L & \text { length scale in the streamwise direction } \\ L_{x}, L_{y} & \text { wavelengths in the } x \text { and } y \text { directions } \\ l_{x}, l_{y} & \text { sizes of the heater in the } x \text { and } y \text { directions } \\ M a & \text { Marangoni number } \\ \boldsymbol{n} & \text { normal vector to the surface } \\ p & \text { pressure } \\ \boldsymbol{q} & \text { local flow rate } \\ r & \text { ratio of } l_{y} / l_{x} \\ T & \text { temperature } \\ \Delta T & \text { temperature difference in the film } \\ \boldsymbol{t} & \text { tangential vector on the surface } \\ t & \text { time } \\ \boldsymbol{u} & \text { velocity vector }\end{array}$

$u \quad$ velocity components in the $x$ direction

$v \quad$ velocity components in the $y$ direction

$w \quad$ velocity components in the $z$ direction

$(x, y, z) \quad$ Cartesian coordinates

Greek symbols

$\alpha \quad$ Newtonian's heat transfer coefficient

r stress tensor

$\gamma \quad$ surface tension variation with temperature

$\epsilon \quad$ small parameter for longwave expansion

$\theta \quad$ incline angle of the substrate

$\rho$ density

$\mu \quad$ dynamic viscosity

$v \quad$ kinematic viscosity

$\kappa \quad$ thermal diffusivity

$\chi \quad$ thermal conductivity

$\sigma \quad$ surface tension

$\omega$ frequency of the disturbance

$\begin{array}{ll}\text { Subscripts } \\ 0 & \text { base state } \\ r, i & \text { real,imaginary part } \\ \infty & \text { ambient gas phase far away from the interface }\end{array}$

profile and wavelength of the most unstable mode at the instability threshold are in qualitative agreement with the experimental results. Kalliadasis et al. [10] studied the same problem as that in Ref. [9] using an integral-boundary-layer approximation of the Navier-Stokes equations. The computations have demonstrated that the free surface develops a bump in the region where the wall temperature gradient is positive. The results of the linear stability of this bump with respect to disturbances in the spanwise direction revealed the existence of both a discrete and an essential spectrum. The essential spectrum is always stable, but the discrete spectrum is unstable beyond a critical Marangoni number for a band of wavenumbers in the spanwise direction. Tiwari et al. [11] studied the stability and transient dynamics of thin liquid films flowing over locally heated surfaces. Because the linearized operators governing the evolution of perturbations are nonnormal due to the spatial nonuniformity of the base state, the authors used a nonmodal, transient analysis to determine the maximum amplification of small perturbations to the film.

Frank [12] has performed a numerical simulation of the 3D structure formation by solving the Navier-Stokes equations using the method of particles for incompressible fluid. The formation of periodic rivulet-like structure has been simulated and compared with the experimental results. More recently, Frank and Kabov [13] experimentally and numerically studied the regular structure formation in a film falling down a vertical plate with a built-in rectangular heater. The authors have studied the dependencies of the critical Marangoni number and the wavelength of the most unstable mode on the Reynolds and Weber numbers. Kabova et al. [14] have investigated the influence of the spanwise and streamwise arrangement of the rectangular heaters on 3D structures of the film surface by solving the nonlinear evolution longwave equation.

In the present work, we investigate the influence of mutual location of heaters on the dynamics of a thin film falling down a locally heated plate. The longwave approximation is used to reduce the Navier-Stokes equations with free-surface boundary conditions to a nonlinear partial differential equation for the evolution of the local height of the free surface. We will study two typical cases, i.e. the heaters with rectangular shapes and with infinite spanwise lengths. We solved the 2D and 3D steady states using Newtonian iteration method for these two cases, respectively. Moreover, we studied the linear stability and nonmodal stability of locally heated films for these two typical cases.

The present paper is organized as follows. In Section 2, the mathematic formulation of the physical model is presented. In Section 3 , the results and discussions are presented on the problem of steady state. In Section 4, the results and discussions is presented on the linear stability analysis and nonmodal stabilities. In Section 5 , we summarize the results and present the conclusions.

\section{Mathematical formulation}

Consider a thin liquid film falling down an inclined substrate with inclination angle $\theta$ with respect to the horizontal direction, as shown in Fig. 1. The coordinate system is constructed with $x$ in the streamwise direction, $y$ the spanwise direction and $z$ normal to the substrate. A heater or a couple of heaters is embedded in the substrate and produces the temperature field $T_{0}(x, y)$ at the plate surface. Far upstream of the heater, the film has constant thickness, $H$. The thickness of the film is denoted by $h(t, x, y)$. The velocity field $\boldsymbol{u}$, temperature $T$ and pressure $p$ of the film are governed by the continuity equation, the Navier-Stokes equation and the energy equation for incompressible Newtonian fluids:

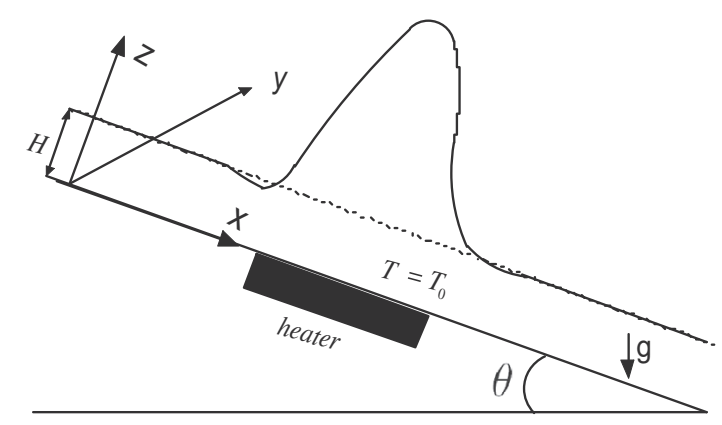

Fig. 1. Sketch of the physical model of a thin film flowing down a locally heated plane. 
$\nabla \cdot \boldsymbol{u}=0$

$\frac{\partial \boldsymbol{u}}{\partial t}+\boldsymbol{u} \cdot \nabla \boldsymbol{u}=-\frac{1}{\rho} \nabla p+v \nabla^{2} \boldsymbol{u}+\mathbf{g}$,

$\frac{\partial T}{\partial t}+\boldsymbol{u} \cdot \nabla T=\kappa \nabla^{2} T$,

where $\rho, v$ and $\kappa$ are the density, kinematic viscosity and thermal diffusivity of the fluid, and $\mathbf{g}$ is gravitational acceleration.

At the substrate surface $(z=0)$, the no-slip and no-penetration boundary conditions are satisfied,

$\boldsymbol{u}(x, y, 0)=0$.

The heater at the substrate surface is assumed to be a good conductor of heat, and produce a temperature distribution at wall as

$T(x, y, 0)=T_{0}(x, y)$.

At the free surface $(z=h(t, x, y))$, the normal and tangential stress balances are

$\boldsymbol{n} \cdot \boldsymbol{\Gamma} \cdot \boldsymbol{n}=\sigma\left(\boldsymbol{\nabla}_{s} \cdot \boldsymbol{n}\right)$,

$\boldsymbol{t} \cdot \boldsymbol{\Gamma} \cdot \boldsymbol{n}=-\boldsymbol{t} \cdot \nabla_{s} \sigma$

where $\boldsymbol{\Gamma}$ is the stress tensor, $\sigma$ is the surface tension, $\nabla_{s}$ is the surface gradient operator, and $\boldsymbol{n}$ and $\boldsymbol{t}$ are the unit vector in the norm and tangent directions at the interface. We assume that the surface tension is linear dependent on the temperature, i.e. $\sigma=\sigma_{0}$ $-\gamma\left(T-T_{S}\right)$, in which $\gamma$ is the surface tension variation with temperature, $\sigma_{0}$ is the surface tension at the reference temperature $T_{s}$.

The boundary condition of the temperature at the free surface is given by Newton's cooling law,

$-\chi \frac{\partial T}{\partial z}=\alpha\left(T-T_{\infty}\right)$,

in which $\chi$ is the thermal conductivity of the liquid and $\alpha$ is the Newton's heat transfer coefficient describing the rate of heat transport from the liquid to the ambient gas phase with temperature $T_{\infty}$ far away from the interface.

The kinematic boundary condition at the interface is

$\frac{\partial h}{\partial t}+\nabla \cdot \mathbf{q}=0$

in which $\boldsymbol{q}=\int_{0}^{h} \boldsymbol{u}[$ ofortt $] \mathrm{d}[$ cfortt $] z$ is the flux.

For the present problem, an appropriate choice of the length scale and the time scale is based on a balance of viscous and gravitational forces [9]. The dimensionless variables (primed) are defined by

$x=L x^{\prime}, \quad y=L y^{\prime}, \quad z=H z^{\prime}$,

$u=\frac{g \cos \theta H^{2}}{v} u^{\prime}, \quad v=\frac{g \cos \theta H^{2}}{v} v^{\prime}, \quad w=\frac{g \cos \theta H^{3}}{v L} w^{\prime}$,

$p-p_{\infty}=\rho g L \cos \theta p^{\prime}, \quad t=\frac{v L}{g \cos \theta H^{2}} t^{\prime}, \quad T-T_{\infty}=\Delta T T^{\prime}$,

where $L$ is the length scale in the streamwise direction over which temperature varies, $p_{\infty}$ is the ambient pressure, $\Delta T$ is the temperature jump at a heater. Applying standard lubrication theory in terms of the parameter $\epsilon=H / L$, after dropping the primes, we obtain the leading-order equations:

$$
\begin{aligned}
& \frac{\partial^{2} u}{\partial z^{2}}-\frac{\partial p}{\partial x}+1=0 \\
& \frac{\partial^{2} v}{\partial z^{2}}-\frac{\partial p}{\partial y}=0 \\
& \frac{\partial p}{\partial z}=0 \\
& \frac{\partial^{2} T}{\partial z^{2}}=0
\end{aligned}
$$

At $z=0$, the boundary conditions are

$u=v=0, T=T_{0}(x, y)$.

At $z=h(t, x, y)$, the boundary conditions are

$\frac{\partial T}{\partial z}+B i T=0, \frac{\partial}{\partial z}\left(\begin{array}{l}u \\ v\end{array}\right)=-M a \nabla T_{i}$,

$p=-\frac{1}{B o} \nabla^{2} h$,

where the Biot number $B i$, the Marangoni number $M a$ and the Bond number are defined as

$B i=\frac{\alpha H}{\chi}, \quad M a=\frac{\gamma \Delta T}{H L \rho g \cos \theta}, \quad B o=\frac{\rho g L^{3} \cos \theta}{H \sigma_{0}}$.

Solving this set of equations, we obtain the interfacial temperature $T_{i}$ and the velocity components in the streamwise and the spanwise directions as

$T_{i}=\frac{T_{0}(x, y)}{1+B i h}$,

$\left(\begin{array}{l}u \\ v\end{array}\right)=-\frac{z^{2}}{2 B o} \nabla \nabla^{2} h-\frac{z^{2}}{2}\left(\begin{array}{l}1 \\ 0\end{array}\right)+z\left(\begin{array}{l}a \\ b\end{array}\right)$,

in which

$\left(\begin{array}{l}a \\ b\end{array}\right)=\frac{h}{B o} \nabla \nabla^{2} h+\left(\begin{array}{l}1 \\ 0\end{array}\right) h-M a \nabla T_{i}$

Integrating $u$ and $v$ from 0 to $h$, we can obtain the expression of $\boldsymbol{q}$ as

$\boldsymbol{q}=\frac{h^{3}}{3 B o} \nabla \nabla^{2} h+\frac{h^{3}}{3}\left(\begin{array}{l}1 \\ 0\end{array}\right)-M a \nabla T_{i} \frac{h^{2}}{2}$

Substituting Eq. (22) into the kinematic relation in Eq. (9), we obtain the evolution equation of the thickness of the film,

$\frac{\partial h}{\partial t}+\nabla \cdot\left(\frac{h^{3}}{3 B o} \nabla \nabla^{2} h-\frac{h^{2}}{2} M a \nabla T_{i}\right)+\frac{1}{3} \frac{\partial h^{3}}{\partial x}=0$.

In the present paper, we will study the influence of the shape and the mutual location of heaters on the dynamics of the thin film. We define $l_{x}$ and $l_{y}$ as the streamwise and the spanwise sizes. For a couple of heaters, we define $d$ as the distance between the centers of heaters. For a heater with a finite streamwise length $\left(l_{x}\right)$ and an infinite spanwise length, the temperature at the plate surface can be prescribed as

$T_{0}(x)=f(x)=0.5\left[\tanh \left(x-l_{x} / 2\right)-\tanh \left(x+l_{x} / 2\right)\right]$.

For a heater with a rectangular shape $\left(l_{x} \times l_{y}\right)$, the temperature at the plate surface can be prescribed as

$$
\begin{aligned}
T_{0}(x, y)= & f(x, y) \\
= & 0.25\left[\tanh \left(x-l_{x} / 2\right)-\tanh \left(x+l_{x} / 2\right)\right][\tanh (y \\
& \left.\left.-l_{y} / 2\right)-\tanh \left(y+l_{y} / 2\right)\right] .
\end{aligned}
$$

For a couple of heaters arranged in the streamwise direction with a distance of $d$, the temperature at the plate surface can be given as

$T_{0}(x, y)=f(x-d, y)+f(x, y)$.

\section{Base state}

The steady base profile is found by seeking solutions of $h(x, y)$. For the case of heaters with infinite spanwise lengths, the temperature profile $T_{0}$ is one dimensional. In this case, the base profile is uninform in the spanwise direction and has the form of $h(x)$. For the case of rectangular heaters, we aim to seek the 3D steady 
profile in the form of $h(x, y)$. Let $\partial_{t} h=0$, we obtain the following nonlinear differential equation for the steady profile of the film

$$
\begin{aligned}
\frac{\partial}{\partial x} & {\left[-\frac{3 M a}{2} \frac{\partial T_{i}}{\partial x} h^{2}+\left(\frac{1}{B o} \frac{\partial}{\partial x} \nabla^{2} h+1\right) h^{3}\right] } \\
& +\frac{\partial}{\partial y}\left[-\frac{3 M a}{2} \frac{\partial T_{i}}{\partial y} h^{2}+\frac{h^{3}}{B o} \frac{\partial}{\partial y} \nabla^{2} h\right] \\
& =0 .
\end{aligned}
$$

This equation is to be solved with the far-field boundary condition $h \rightarrow 1$ as $x \rightarrow \pm \infty$. We have now introduced new lengthscales, $L_{x}$ and $L_{y}$, the $x, y$ periods of the array. For 2D problem, in the limit of infinite $L_{x} \rightarrow \infty$ we recover the case of a single or a pair of heaters. The periodic solutions of Eq. (27) is obtained by a parametric continuation method combined with Fourier expansions. We start with the flat-film solution at $M a=0$ for fixed $B o$ and $B i$ by increasing $M a$ to the desired value. In each step of increasing $M a$, a Newtonian iteration method is used to solve the nonlinear equation for $h$.

In Fig. 2, base profiles are plotted for a thin film heated by a single heater and a couple of heaters with infinite spanwise lengths. As shown in Fig. 2, at the upstream edge of the heaters, the Marangoni stress induced by temperature gradient opposes the flow of the film, resulting in the thickening of film to maintain a constant flow rate and forming pronounced thermocapillary ridges. In the case of only one heater, a bump structure has been formed in this figure. In the case of a couple of heaters, for $d=2$ two bumps has been formed at the upstream edge of the two heaters. The amplitude of the downstream bump is smaller than that of the upstream one. As the distance increases to $d=4$, it is found that the two bumps almost have the same height. These bumps can be unstable to infinitesimal disturbance. We will study the influence of the mutual location on the stability of the film in the next section.

Being similar to films heated by heaters with infinite spanwise length, for films heated by rectangular heaters a steady state of the interfacial deformation exists. However, for thin films heated by rectangular heaters, the base profiles are three dimensional. Fig. 3 displays the base profiles for films heated by a single rectangular heater. In each figure in Fig. 3, the film thickens in the upper stream direction near the heater. Two lateral waves are formed near the lateral sides of the heater and extend to a distance much

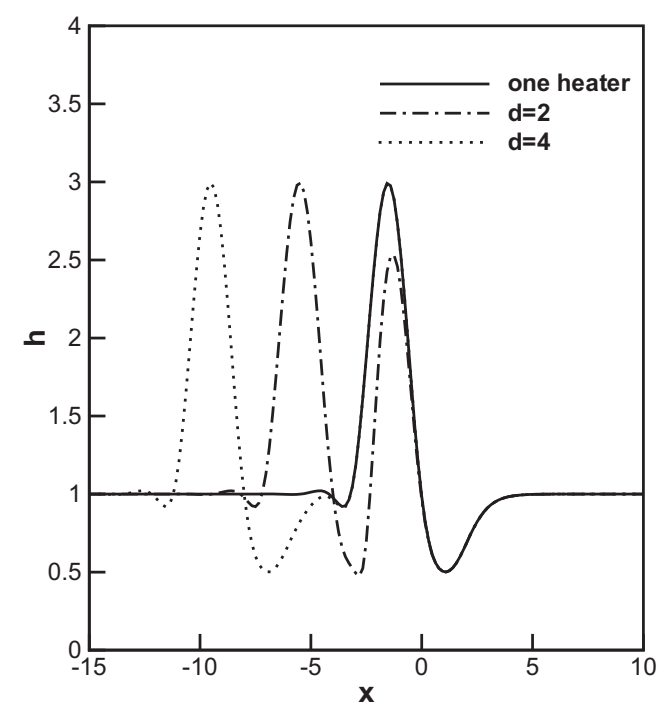

Fig. 2. The film profiles of the steady solutions for films heated by heaters with infinite spanwise length and streamwise length $l_{x}=2$. The other parameters are $B i=0, M a=5.0$ and $B o=10$. longer than the heater length in the downstream direction. The film thickens in the lateral waves and thins in the inner regions near the lateral waves. As shown in Fig. 3(a) for a rectangular heater with $l_{x}=l_{y}=2$, only one thinning wave is present in the inner region between the two lateral waves in the downstream direction. The position of the minimum film thickness occurs in the downstream direction near the heater. With the increase of the spanwise length of the heater, as shown in Fig. 3(b) and (c) for $l_{y}=8$ and $l_{y}=16$ two thinning waves appear in the inner region. The position of the minimum film thickness occurs in the inner region near the lateral of the heaters in the downstream direction.

In order to know the effect of mutual location of heaters on the structure of steady profile, we plot in Fig. 4 the contour of thickness of the film heated by a couple of heaters with distance $d=5$. In Fig. 4(a) for heaters with $l_{x}=l_{y}=2$, two lateral waves are formed from the first heater and extend to a long distance in the downstream direction. The film thickens in the upstream direction near the first heater and in the lateral waves. The position of the minimum thickness of film occurs in the downstream direction near the second heater. In Fig. 4(b) and (c) for heaters with wider spanwise lengths, the film thickens in the upstream directions near the two heaters. Being different from the case heated by a single heaters, two lateral waves are formed from the second heater in Fig. 4(b) and (c). In Fig. 4(a)-(c), the position of the minimum film thickness always occurs in the downstream direction near the second heater.

In order to know the influence of the shape of heater on the rupture of the film, we plot in Fig. 5 the minimum thickness of the film at various Marangoni numbers for various ratios of $r=l_{y} / l_{x}$. In Fig. 5(a) for films heated by one heater, the minimum thickness decreases with the increase of the Marangoni number. As the Marangoni number approaches to a certain value $\left(M a_{c}\right)$, the minimum thickness decreases dramatically to zero. We should note that $M a_{c}$ here is not is related to the threshold for instability. Here $M a_{c}$ has been redefined as the value at which the minimum film thickness tends to zero. Unless stated otherwise $M a_{c}$ means the threshold for instability. For $r=1$, the Maragoni number at which film rupture occurs is slightly greater than 1 . In this figure, $M a_{c}$ increases with the increase of $r$. As $r$ increase to 8, film rupture occurs at $M a_{c} \approx 3$. This result shows that increasing the spanwise length of the heater can inhibit the rupture of the film. In Fig. 5(b), the curves of minimum thickness of films heated by a couple of heaters are plotted for various $r$. Comparing the curves in Fig. 5(b) and (a), we find that film rupture occurs at a lower Marangoni number for a film heated by two heaters than by one heater.

The Biot number is an important parameter to influence the rupture and stability of a locally heated film. In Fig. 6, we plot the curves of the minimum thickness at different Biot numbers. For each case in Fig. 6, the minimum thickness increases significantly with the Biot number when $B i$ is small. For larger Boit numbers, the minimum thickness gradually increases with the increase of $B i$. This result indicates that the increase of the Biot number will inhibit rupture of the film.

\section{Linear stability analysis}

For films heated by heater with finite spanwise length, the thickness of the basic state displays a 3D structure. To study the linear stability of 3D steady state to infinitesimal perturbations, a small perturbation to the film thickness is imposed,

$h(x, y, t)=h_{0}(x, y)+\varepsilon h_{1}(x, y, t)$,

with $\varepsilon \ll 1$. Here, $h_{0}(x, y)$ denote the film thickness of the steady base state.

At last, we obtain the linearized equation 

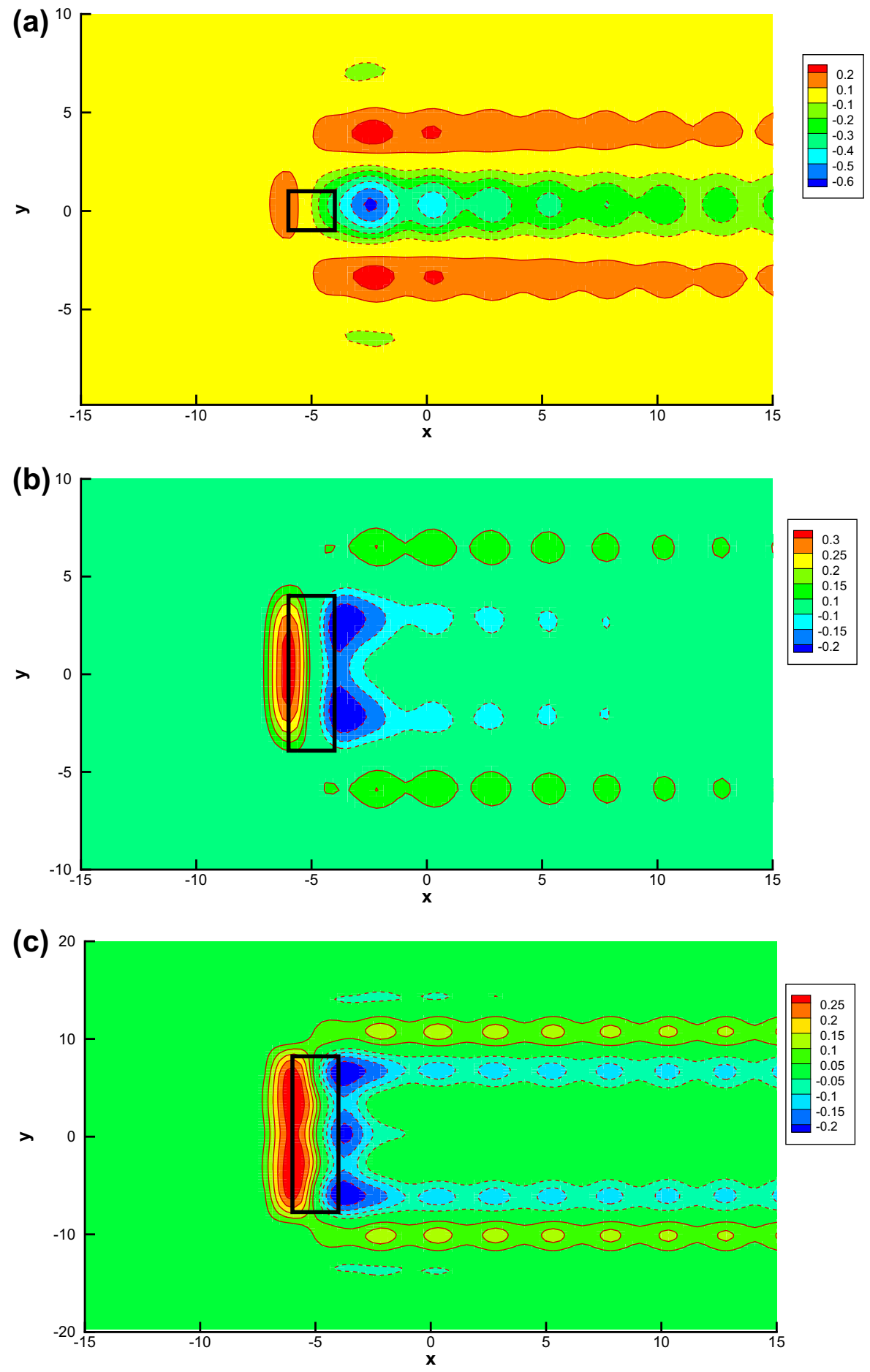

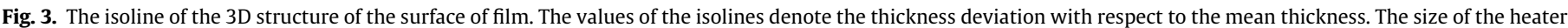
is (a) $l_{x}=2, l_{y}=2$ (b) $l_{x}=2, l_{y}=8$, (c) $l_{x}=2, l_{y}=16$. The other parameters are $M a=1.0, B i=0$ and $B o=10$. Wavelengths are $L_{x}=L y=40$.

$$
\begin{aligned}
\frac{\partial}{\partial t} & h_{1}+\partial_{x}\left\{-\frac{1}{2} M a\left[h_{0}^{2}\left(\partial_{x} T_{i}\right)^{\prime}+\left(\partial_{x} T_{i}\right)_{0} 2 h_{0} h_{1}\right]\right. \\
& \left.+\frac{1}{3 B o}\left[\left(\partial_{x} \nabla^{2} h_{0}\right) 3 h_{0}^{2} h_{1}+h_{0}^{3} \partial_{x} \nabla^{2} h_{1}\right]+h_{0}^{2} h_{1}\right\} \\
& +\partial_{y}\left\{-\frac{1}{2} M a\left[h_{0}^{2}\left(\partial_{y} T_{i}\right)^{\prime}+\left(\partial_{y} T_{i}\right)_{0} 2 h_{0} h_{1}\right]\right. \\
& \left.+\frac{1}{3 B o}\left[\left(\partial_{y} \nabla^{2} h_{0}\right) 3 h_{0}^{2} h_{1}+h_{0}^{3} \partial_{y} \nabla^{2} h_{1}\right]\right\}=0,
\end{aligned}
$$

in which

$$
\begin{aligned}
& \left(\frac{\partial T_{i}}{\partial x}\right)^{\prime}=-\frac{\partial_{x} T_{0}}{\left(1+B i h_{0}\right)^{2}} B i h_{1}-\frac{T_{0} B i \partial_{x} h_{1}}{\left(1+B i h_{0}\right)^{2}}+\frac{2 T_{0} B i \partial_{x} h_{0}}{\left(1+B i h_{0}\right)^{3}} B i h_{1}, \\
& \left(\frac{\partial T_{i}}{\partial y}\right)^{\prime}=-\frac{\partial_{y} T_{0}}{\left(1+B i h_{0}\right)^{2}} B i h_{1}-\frac{T_{0} B i \partial_{y} h_{1}}{\left(1+B i h_{0}\right)^{2}}+\frac{2 T_{0} B i \partial_{y} h_{0}}{\left(1+B i h_{0}\right)^{3}} B i h_{1} .
\end{aligned}
$$

For heaters with finite/infinite spanwise lengths, the steady state is three-dimensional/two-dimensional. Assuming an exponential time dependence for $h_{1}$, we use a perturbation of the form

$$
h_{1}(x, y, t)=\varepsilon \hat{H}(x, y) e^{-i \omega t},
$$



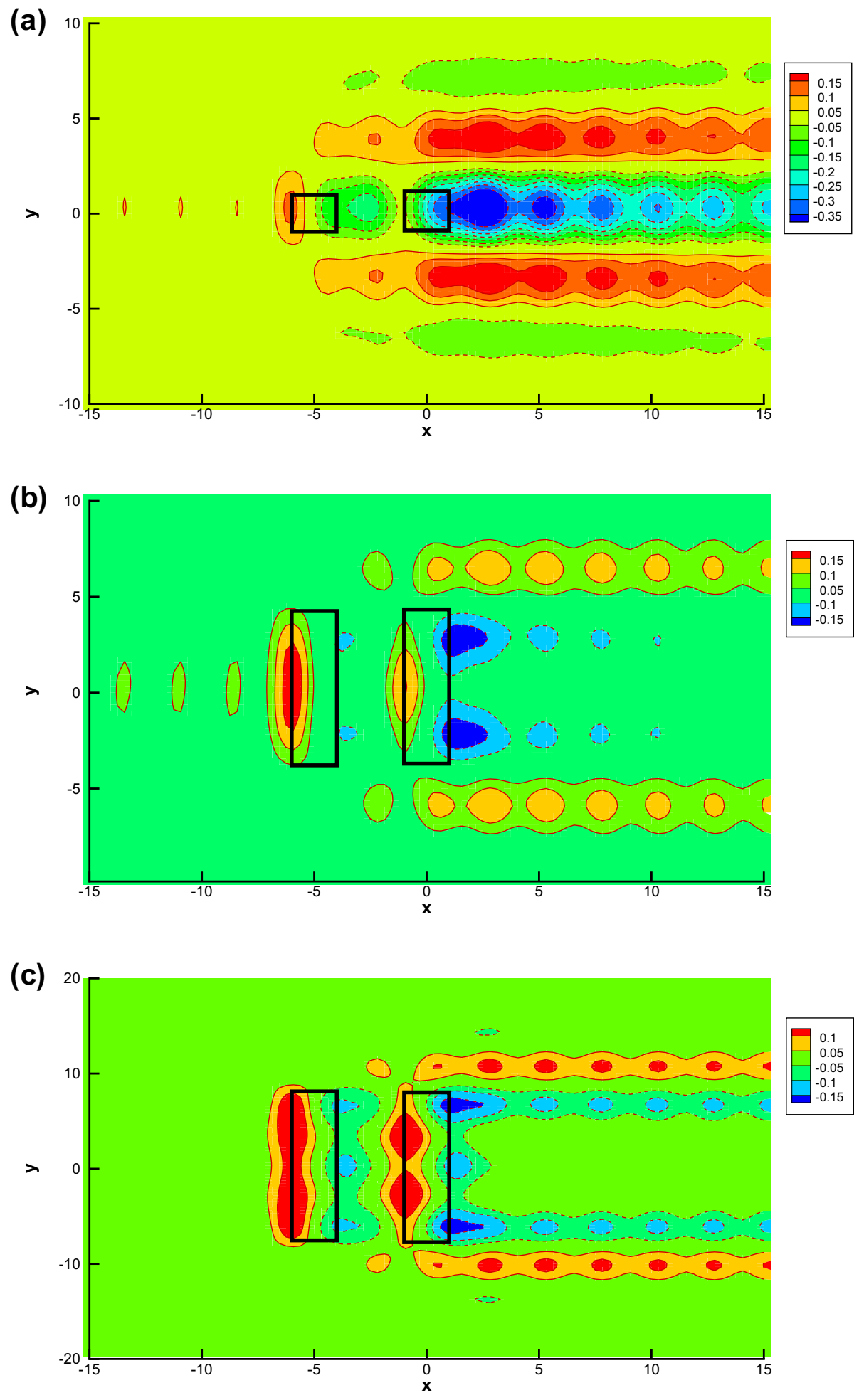

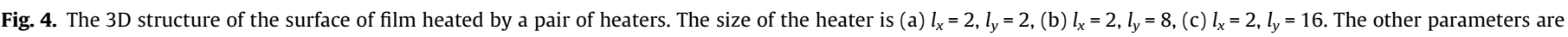
$M a=0.5, B i=0$ and $B o=10$. Wavelengths are $L_{x}=L y=40$.

for 3D stability problem, and

$h_{1}(x, y, t)=\varepsilon \hat{H}(x) e^{-i(\omega t-k y)}$,

for 2D stability problem, where $\omega$ is the complex frequency and $k$ is the spanwise wavenumber. Substituting Eq. (32) into Eq. (29) yields the eigenvalue problem in the form of

$\omega \hat{H}=\mathscr{L} \hat{H}$, where $\mathscr{L}$ is a linear operator. In order to study the stability, we assume the periods of array in $x$ and $y$ directions are $L_{x}$ and $L_{y}$. The eigenvalue problem is solved numerically by a Fourier collocation method. $\hat{H}$ is expanded by a global Fourier spectral series in the streamwise and spanwise coordinates $x, y$

$\hat{H}(x, y)=\sum_{m=M n=N}^{M} \sum_{m, n}^{N} \widetilde{H} e^{\frac{i 2 \pi m x}{L_{x}}} e^{\frac{i 2 \pi n y}{L_{y}}}$. 
(a)

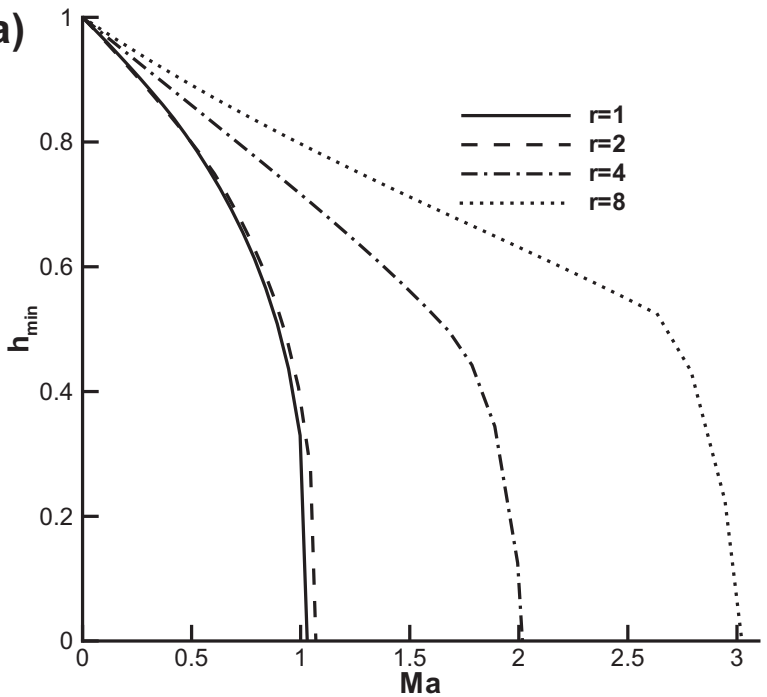

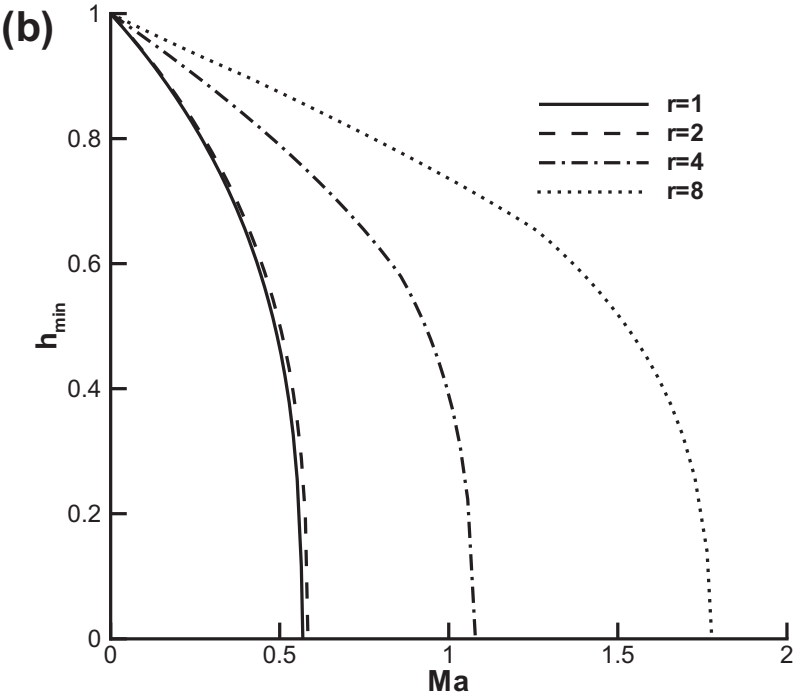

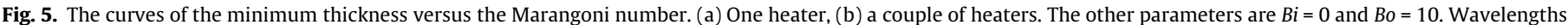
are $L_{x}=L y=40$.
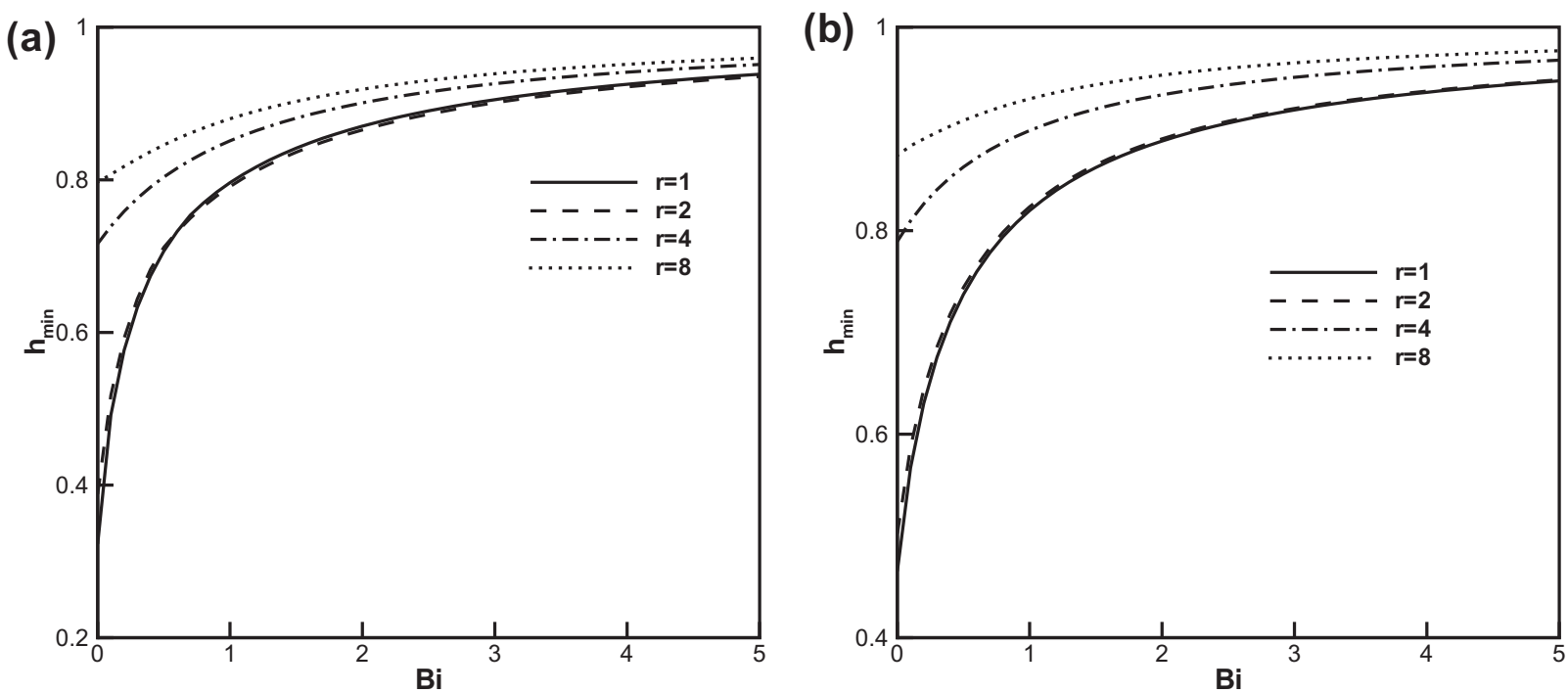

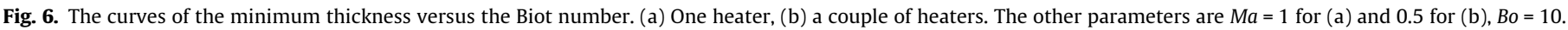
Wavelengths are $L_{x}=L y=40$.

We then substitute Eq. (35) into Eq. (34) and apply the resulting equations at $2 M$ and $4 M N$ collocation points for $2 \mathrm{D}$ and $3 \mathrm{D}$ problems. Therefore, we obtain a set of $2 M \times 2 M$ and $4 M N \times 4 M N$ eigenvalue problems for $2 \mathrm{D}$ and $3 \mathrm{D}$ cases. For more detail about the Fourier collocation method, we refer the readers to the book by Canuto et al. [15].

To know the stability of the disturbances, we will solve all the eigenvalues of $\omega$. The growth rate of the amplitude of a given mode is determined by $\omega_{i}=\operatorname{Im}(\omega)$ : The amplitude of disturbance will grow exponentially in time if $\omega_{i}>0$ and will decay exponentially if $\omega_{i}<0$. The eigenvalue with the largest imaginary part of $\omega$, corresponds to the most unstable mode.

In order to know the effect of mutual location of the heaters on the stability of the films heated by heaters with infinite spanwise length, in Fig. 7 we plot the curves of the dispersion relation for a film heated by one heater and a pair of heaters with different distances $d$. As shown in Fig. 7(a) for $B i=0$, each curve consists of two branches. The left branch is an oscillatory branch with a non-zero real part of $\omega$. The right branch is a static branch with a zero real part of $\omega$, which corresponds to the rivulet instability. For a film heated by two heaters with $d=1$, the dispersion curve is almost indistinguishable to that of a film heated by one heater. With the increase of $d$, it is found that both the oscillatory and the static branches become more unstable. Moreover, the oscillatory branch extends to the shortwave region with the increase of $d$. In Fig. 7(b), oscillatory branch does not appear in the cases of one heater and $d=1,2$. As $d$ increase to 4 , the oscillatory branch begins to appear in the longwave region. Being similar to the curves in Fig. 7(a) for $B i=0$, the timegrowth rate increases with the increase of $d$.

In Fig. 8, we plot the curves of the timegrowth rate of the most unstable mode versus the Marangoni number for the case of $B o=10, B i=0.1$. The period of the heater array is $L_{x}=20$. For period located heaters, the growth rate is always great than zero for all Marangoni numbers. It is found that at large Ma the increase of $\omega_{i}$ is proportional to the increase of $M a$. At large $M a$, it is found that the time growth rate is very close to the case of $L_{x} \longrightarrow \infty$. This result 

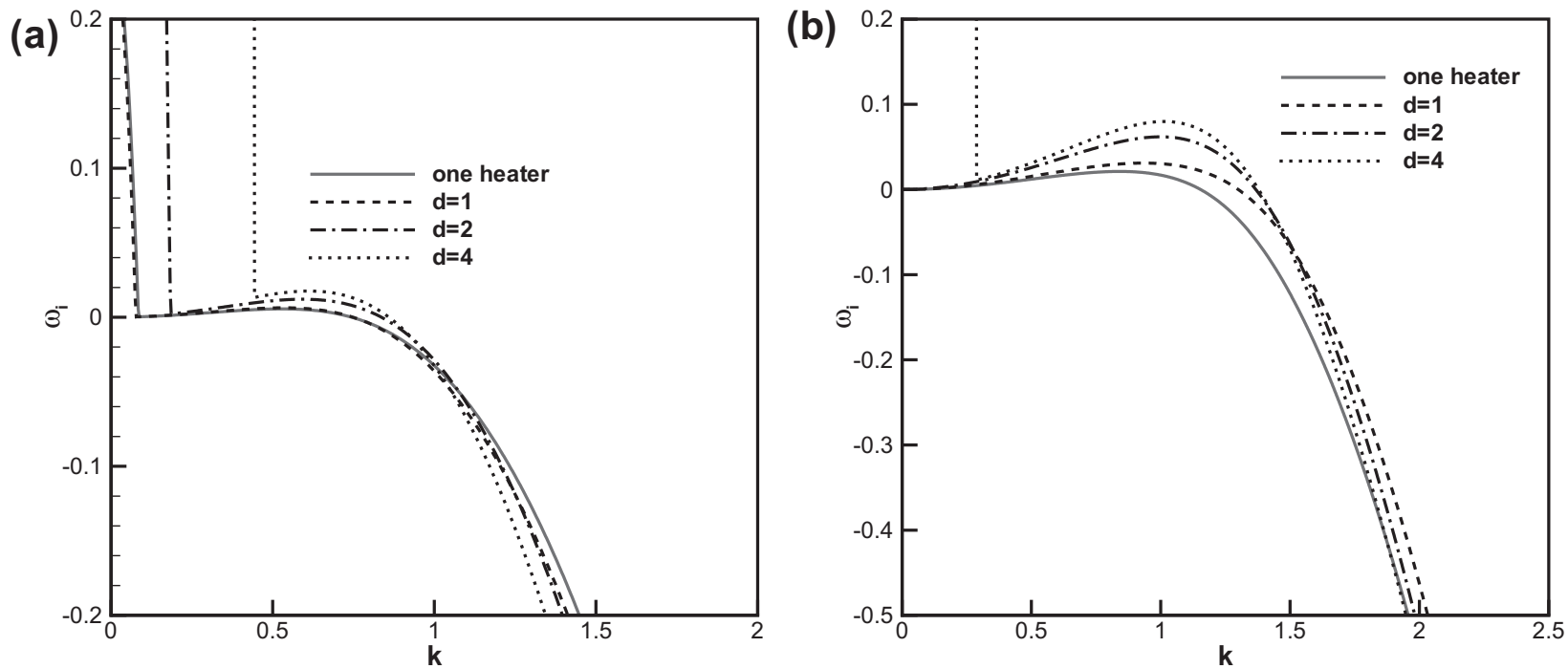

Fig. 7. Dispersion relations of the growth rate $\omega_{i}$ versus the wavenumber $k$. (a) For $M a=5.0, B i=0, B o=10$; (b) for $M a=5.0, B i=0.1, B o=10$. Wavelength $L_{x}=20, l_{x}=2$.

is consistent to the result in Ref. [9]. For $L_{x} \longrightarrow \infty$, it is found that the critical value of the Marangoni number is in insensitive to the distance between the heaters. The critical Marangoni number for instability is $M a_{c} \approx 6$ for all cases in Fig. 8 .

We have also solved the eigenvalue problem for films heated by heaters with finite spanwise lengths. The results show that there is no unstable mode for the 3D stability problem. This means the 3D steady state is always stable in the framework of modal stability analysis.

\section{Nonmodal stability analysis}

For locally heated films, the dynamics of infinitesimal disturbances can be described by a linear operator $\frac{d}{d t} h_{1}=-i \mathscr{L} h_{1}$. When the 2D base states are spatially nonuniform, thin liquid films with thermocapillary stresses give rise to non-normal linear operators with eigenvectors that are not orthogonal. For a non-normal linear system, transient amplification due to the interaction of the nonorthogonal eigenvector could lead to a large growth of finiteamplitude disturbance at short time. For a locally heated film, the disturbance that undergoes a substantial amplification at short time

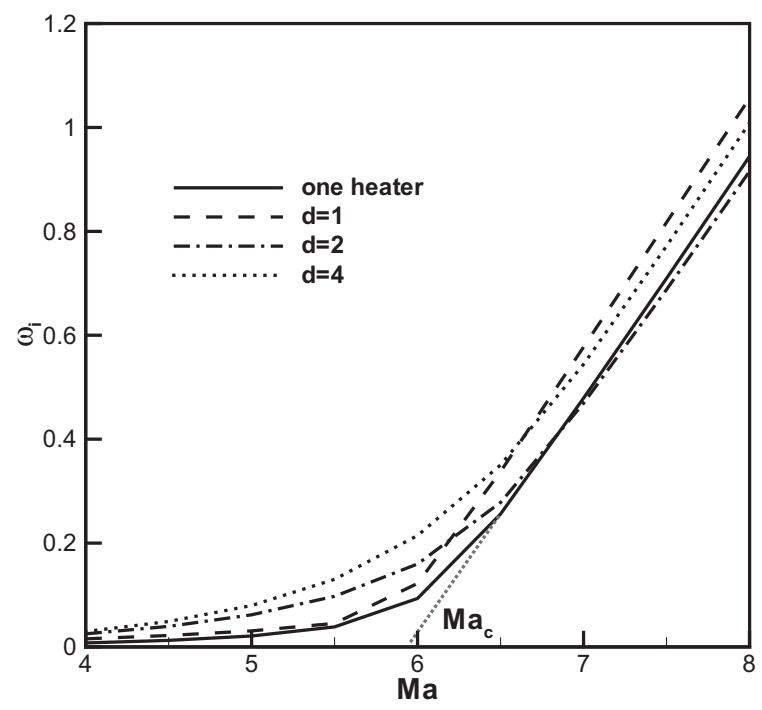

Fig. 8. The growth rate for the most unstable mode, $\omega_{i}$, versus Marangoni number with $B_{o}=10, B_{i}=0.1$. Wavelength $L_{x}=20, l_{x}=2$. may lead to rupture of the film. So, understanding the short-time behavior of the disturbance may be of crucial importance to stabilize the film. Tiwari et al. [11] have studied the transient amplification using nonmodal analysis for the 2D problem of thin liquid films flowing over a heater. In the present paper, we will study the transient amplification for thin liquid films heated by a couple of heaters, and examine the influence of the arrangement of heaters on the nonmodal stability. Moreover, we will study the nonmodal stability of disturbance for the 3D problem.

In the nonmodal theory, two types of problems are of particular interest, i.e., response to external excitations and transient energy growth of initial conditions. For the former, the norm of resolvent denotes the maximum amplification of external excitations; for the latter, the growth function $G(t)$ identifies the optimal growth of energy at time $t$. The concept of numerical range can be used to link the operator to the initial energy growth. The numerical range is defined as

$W(\mathbf{A})=\left\{\mathbf{x}^{*} \mathbf{A} \mathbf{x}: \mathbf{x} \in \mathbb{C}^{N},\|\mathbf{x}\|=1\right\}$,

in which $\mathbf{A} \in \mathbb{C}^{N \times N}$. The numerical abscissa of $\mathbf{A}$ is defined as:

$\omega(\mathbf{A})=\sup _{z \in W(\mathbf{A})} \operatorname{Re}(z)=\lim _{t \rightarrow 0} \frac{\mathrm{d}}{\mathrm{d} t}\left\|e^{\mathbf{A} t}\right\|$.

The main application of numerical range is to the analysis of energy growth for initial-value problems. The numerical abscissa of $-i \mathscr{L}$ corresponds to the initial growth rate of the energy growth function.

We begin with the responses to external excitations. Suppose a linear system is driven by a harmonic signal $\boldsymbol{q}_{\text {in }}$ with a frequency of $\omega$. Then the response $\boldsymbol{q}_{\text {out }}$ and the input signal $\boldsymbol{q}_{\text {in }}$ satisfy the equation

$\frac{\partial}{\partial t} \boldsymbol{q}_{\text {out }}=-i \mathscr{L} \mathbf{q}_{\text {out }}+\boldsymbol{q}_{\text {in }}$.

From Eq. (38), we obtain the response $\boldsymbol{q}_{\text {out }}$ in the form of

$\mathbf{q}_{\text {out }}(x, y, z, t)=i(\omega \mathscr{I}-\mathscr{L})^{-1} \boldsymbol{q}_{\text {in }}(x, y, z)$.

Here $\mathscr{I}$ is the identical matrix. The solution operator $(\omega \mathscr{I}-\mathscr{L})^{-1}$ is known as the resolvent.

We denote the maximum amplification of a disturbance at frequency $\omega$ by $\mathscr{R}(\omega)$. $\mathscr{R}(\omega)$ is equal to the norm of the resolvent and expressed as

$\mathscr{R}(\omega)=\sup _{\boldsymbol{q}_{\text {in }} \neq 0} \frac{\left\|\boldsymbol{q}_{\text {out }}\right\|}{\left\|\boldsymbol{q}_{\text {in }}\right\|}=\left\|(\omega \mathscr{I}-\mathscr{L})^{-1}\right\|$. 
(a)

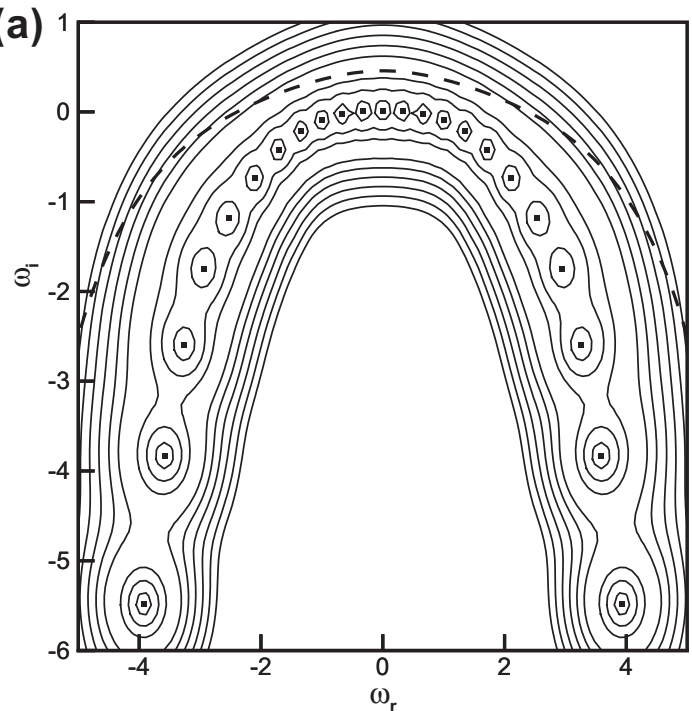

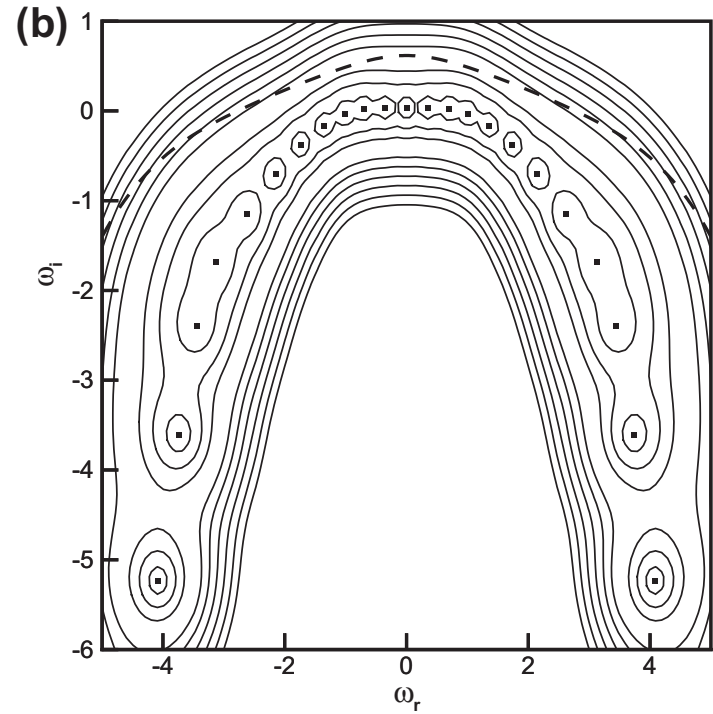

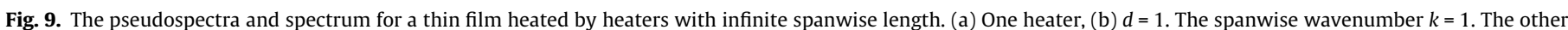

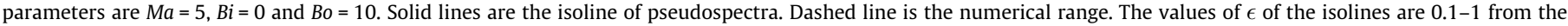
innermost to the outermost. Wavelength $L_{x}=20, l_{x}=2$.

Here $\|\cdot\|$ denotes a norm on $\mathbb{C}^{N}$, "sup" denotes the maximum. An eigenvalue of $\mathscr{L}$ is a number $\omega$ such that $\left\|(\omega \mathscr{I}-\mathscr{L})^{-1}\right\| \rightarrow \infty$. Generalizing this result leads naturally to the definition of " $\epsilon-$ pseudospectrum". For any $\epsilon \geqslant 0$, the " $\epsilon$-pseudospectrum" of $\mathscr{L}$ is defined as

$$
\Lambda_{\epsilon}(\mathscr{L})=\left\{\omega \in \mathbb{C}:\left\|(\omega \mathscr{I}-\mathscr{L})^{-1}\right\| \geqslant \epsilon^{-1}\right\} .
$$

The resolvent norm represents the amplification of response to external forcing.

The behavior of a non-normal operator depends on not solely the eigenvalues, but the structure of $\epsilon$-pseudospectra. In Fig. 9, we present the spectrum and pseudospectra for films heated by heaters with infinite spanwise length. In Fig. 9(a) and (b), the structures of the spectrum and pseudospectra are qualitatively similar. The most amplified mode is static and locates in the unstable half plane. The other modes are oscillatory and stable. The curve of the boundary of numerical range enters the unstable half plane. This means the disturbance has a potential to be substantially amplified. In the complex $\omega$ plane, we are particularly interested in the resonance along the real axis because it corresponds to external excitations at real frequencies. In Fig. 9(a) and (b), the isolines

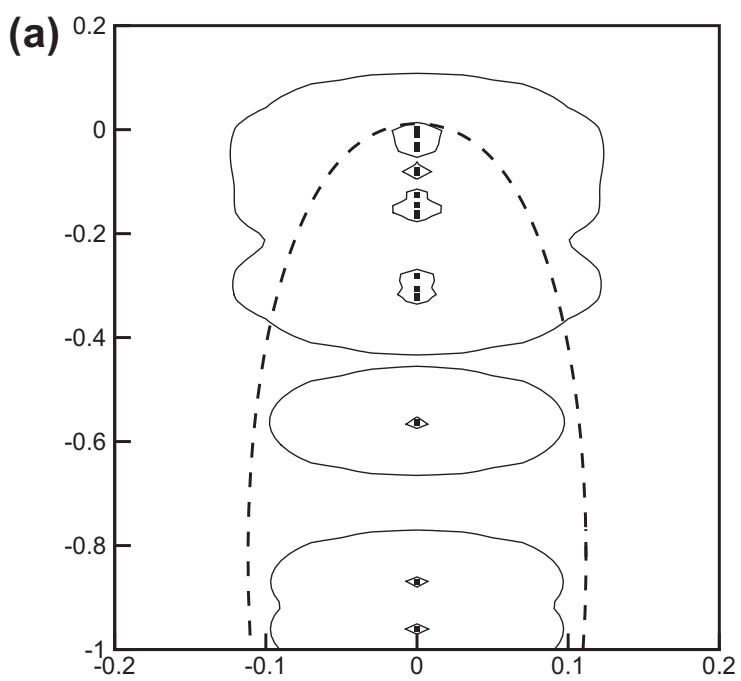

of the pseudospectra show that the external forcing is amplified the most in the low frequency limit of $\omega=0$, and with the increase of $|\omega|$ the amplification decreases.

In Fig. 10, we present the spectrum and pseudospectra for films heated by heaters with finite spanwise lengths. For 3D steady state, all the eigenvalues are stable and most of them locate in the imaginary axis. The boundary of the numerical range only slightly enters the unstable half plane. This means that the transient growth of the disturbance is weak.

Now we examine the problem of response to initial conditions. The general solution to $\frac{\mathrm{d}}{\mathrm{d} t} h_{1}=-i \mathscr{L} h_{1}$ is $h_{1}(t)=\exp (-i \mathscr{L} t) h_{1}(0)$. The maximum amplification of an initial perturbation over the time interval $t$ is the given by

$G(t)=\sup _{h(0) \neq 0} \frac{\left\|h_{1}(t)\right\|}{\left\|h_{1}(0)\right\|}=\|\exp (-i \mathscr{L} t)\|$.

In Fig. 11, the curves of the transient energy growth are plotted for films heated by heaters with infinite spanwise lengths. For $M a=5$, $B i=0.1$ and $B o=10$, we have shown that the film is unstable. So, disturbances of the most unstable mode will grow exponentially

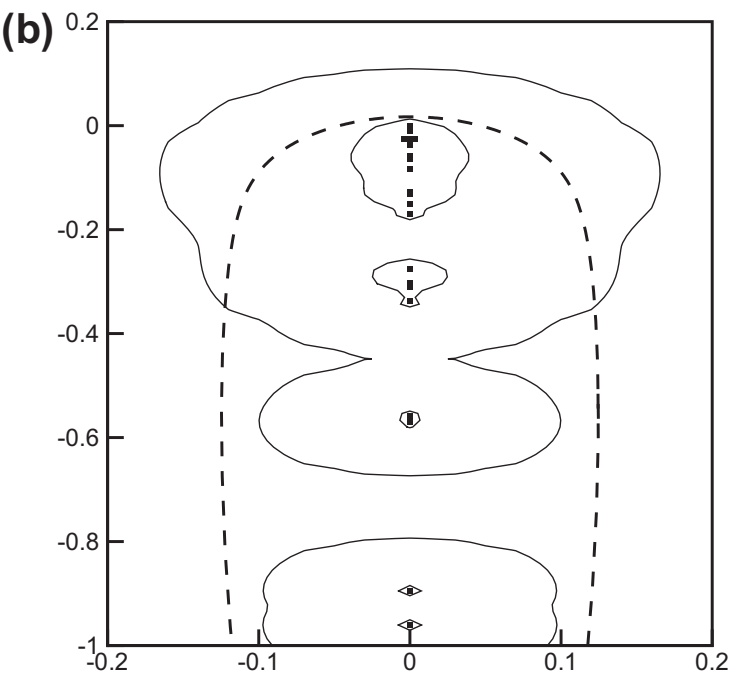

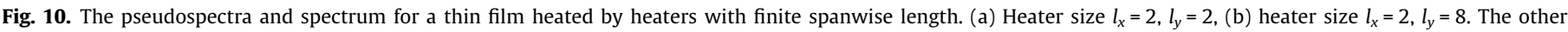
parameters are $M a=1$ for (a) and 1.8 for (b), $B i=0, B o=10$. Wavelength $L_{x}=40, L_{x}=40$. 


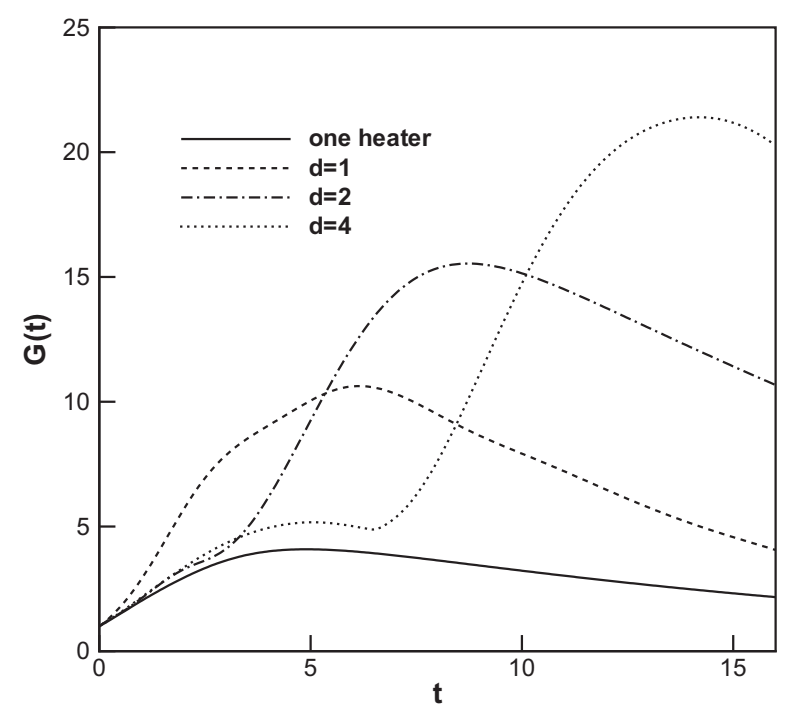

Fig. 11. The transient energy growth of optimal disturbance with $k=1.014$ for a thin film heated by heaters with infinite spanwise length. The other parameters are $M a=5, B i=0.1$ and $B o=10$. Wavelength $L_{x}=20, l_{x}=2$.

with time. However, in Fig. 10 each curve of the optimal disturbance experiences an obvious transient growth before the exponential growth. This means the transient growth could result in the rupture of the film. As shown in this figure, with the increase of the distance between two heaters, both the transient growth and the optimal time increase.

We also examine the transient growth for films heated by heaters with finite spanwise lengths. In this case, the results show that the transient growth is very weak for the 3D problem. For films heated by heaters with finite spanwise lengths, the 3D steady state is a coherent structure. So, in this case the rupture of the film is mainly determined by the structure of 3D steady state.

\section{Conclusions}

In the present paper, the effects of mutual location and the shape of the heaters on the dynamics of thin films flowing over locally heated plates have been examined. We focused on the influence of heaters on the steady state and the stability of the film. For films heated by one heater or a couple of heaters with infinite spanwise wavelength, a 2D steady state exists. This 2D steady state can be unstable when the Marangoni number exceeds the critical value $M a_{c}$. For films heated by heater array with the period of $L_{\chi}$, the timegrowth rate is always positive at $M a>0$. As $L_{x} \rightarrow 0$, the critical Marangoni number $M a \approx 6$ for $B i=0.1$ and $B o=10$. The curves of the timegrowth rate consist of two branches. The left branch is an oscillatory branch, and the right branch is a static branch, which corresponds to the rivulet instability. With the increase of the distance between two heaters, both the oscillatory branch and the static branch become more unstable.

For films heated by heaters with finite spanwise lengths, a 3D steady state of the interfacial deformation exists. We have shown that this 3D steady profile is always stable with respect to infinitesimal disturbances. So, the rupture of film is only determined by the
3D steady state. For a film heated by a single heater, it thickens in the upper stream direction near the heater. Two lateral waves are formed near the lateral sides of the heater, and the film thickens in the lateral waves and thins in the inner regions near the lateral waves. The position of the minimum film thickness occurs in the downstream direction near the heater. For films heated by a couple of heaters, the position of the minimum film thickness always occurs in the downstream direction near the second heater. In this case, the minimum thickness decreases with the increase of the Marangoni number.

We have also studied the nonmodal stability of films heated by one heater and a couple of heaters. For films heated by heaters with infinite spanwise lengths, a substantial transient energy growth is found for disturbances with finite amplitude. However, for films heated by heaters with finite spanwise lengths, the transient growth is very weak. So, the $3 \mathrm{D}$ state is stable in the framework of both the modal and the nonmodal theories. This means that the 3D steady state is a coherent structure for films heated by heaters with finite spanwise lengths.

\section{Acknowledgments}

The authors gratefully acknowledge support of this work by the European Space Agency (HEAT TRANSFER PRODEX Programme, Belgium). R.L. acknowledges support by Belgian Federal Science Policy Office and National Natural Science Foundation of China (Grant No. 11102211).

\section{References}

[1] S.J. VanHook, M.F. Schatz, J.B. Swift, W.D. McCormick, H.L. Swinney, Longwavelength surface-tension-driven Bénard convection: experiment and theory, J. Fluid Mech. 345 (1997) 45-78.

[2] J.R.A. Pearson, On convection cells induced by surface tension, J. Fluid Mech. 4 (1958) 489-500.

[3] R. Mahajan, C. Chiu, G. Chrysler, Cooling a microprocessor chip, Proc. IEEE 94 (8) (2006) 1476-1486.

[4] O.A. Kabov, Heat transfer from a heater with a small linear dimension to a free falling liquid film, in: Proceedings of First Russian National Conference on Heat Transfer, vol. 6, 1994, pp. 90-95.

[5] O.A. Kabov, I.V. Marchuk, V.M. Chupin, Thermal imaging study of the liquid film flowing on a vertical surface with local heat source, Russ. J. Eng. Thermophys. 6 (1996) 105-138.

[6] O.A. Kabov, Formation of regular structures in a falling liquid film upon local heating, Thermophys. Aeromech. 5 (1998) 547-551.

[7] O.A. Kabov, I.V.Marchuk, A.V. Muzykantov, J.C. Legros, E. Istasse, J.L. Dewandel, Regular structures in locally heated falling liquid films, in: Proceedings of Second International Symposium on Two-Phase Flow Modelling and Experimentation, Pisa, vol. 2, 1999, pp. 1225-1233.

[8] O.A. Kabov, Interfacial thermal fluid phenomena in thin liquid films, Int. J. Emerg. Multidiscip. Fluid Sci. 2 (2010) 87-121.

[9] J.M. Skotheim, U. Thiele, B. Scheid, On the instability of a falling film due to localized heating, J. Fluid Mech. 475 (2003) 1-19.

[10] S. Kalliadasis, A. Kiyashko, E.A. Demekhin, Marangoni instability of a thin liquid film heated from below by a local heat source, J. Fluid Mech. 475 (2003) 377-408.

[11] N. Tiwari, Z. Mester, J.M. Davis, Stability and transient dynamics of thin liquid films flowing over locally heated surfaces, Phys. Rev. E 76 (1-14) (2007) 056306.

[12] A.M. Frank, 3D numerical simulation of regular structure formation in a locally heated falling film, Eur. J. Mech. B Fluids 22 (2003) 445-471.

[13] A.M. Frank, O.A. Kabov, Thermocapillary structure formation in a falling film: experiment and calculations, Phys. Fluids 18 (1-10) (2006) 032107.

[14] Y.O. Kabova, V.V. Kuznetsov, O.A. Kabov, The effect of mutual location of heaters on the falling film dynamics, Microgravity Sci. Technol. 19 (2007) 5356.

[15] C. Canuto, M.Y. Hussaini, A. Quarteroni, T.A. Zang, Spectral Method in Fluid Dynamics, Springer, 1993. 